\title{
Institutions as context-sensitive control superstructures for firms
}

\author{
Prateek Goorha* \\ Research Fellow, School of Economics, Finance and Marketing, RMIT University, Melbourne, Australia
}

This paper addresses the lack of context-sensitivity in institutional analysis by introducing control process theory. It suggests that institutions can be seen as control process superstructures that provide a specific environment within which control action is undertaken for a principal-agent relationship. This view is not meant to be a sensationalist departure in opinion from the analysis of institutions or on the nature of the firm but, rather, a simple manner to understand the connections and relevance contained therein within an overarching and intuitive framework that emphasizes contextual sensitivity. The paper also suggests how this approach informs the nature and type of control action available to a principal within an instantiated institution and, further, how it allows the consideration of soft knowledge in devising control action.

Keywords: control process theory, nature of the firm, contextualized institutions, principalagent model, fuzzy information

JEL codes: $D 02, L 16, L 22, P 15, E 02$

\section{INTRODUCTION}

Certain nuances that arise in the characterization of institutions across different contexts are lost when they are generalized in comparisons. While this may well be necessary in studying their function and mechanism, these lost nuances are then liable to be held as having being critical in explaining observed differences in outcomes across institutions. That such context sensitivity 'matters' is a fundamental premise of this paper. At a macro level it may, for example, help explain the differences in performance across two countries of institutions that were designed with the same intent, and, at a more disaggregated level, it may perhaps shed light on how a multinational might arrange ownership differently in different countries. ${ }^{1}$

The nature of this context sensitivity is, of course, itself contentious. While, conveniently, the uniqueness of the context can be considered to be of a social, political, geographic or economic nature, a significant issue to scholars is whether the nature of certain contextual conditions fundamentally impacts other social outcomes. Examples of such endeavors might include questions about whether certain political

* I acknowledge, with sincere gratitude, the suggestions made by Randy Silvers and the anonymous reviewers at EJEEP. I especially wish to thank the managing editor of this journal, Torsten Niechoj, for his helpful suggestions and tireless efforts throughout the review process. 1. As examples, see Rodrik (2008) and Che/Facchini (2009).

Received 12 March 2012, accepted 11 July 2013 
institutions are necessary for economic growth, ${ }^{2}$ whether social norms and traditions predict the effectiveness of formal institutions, ${ }^{3}$ whether certain legal traditions are more likely to give better social outcomes, ${ }^{4}$ whether geography has an overarching influence over socioeconomic outcomes, ${ }^{5}$ and whether religion is crucial to the development of certain political institutions. ${ }^{6}$

The framework we present in this paper is not intended as an all-encompassing methodology for the analysis of all such contexts and for any institution, but, rather, a manner of characterizing the idea of context sensitivity. We deliberately embed firms within an institutional superstructure in the paper so as to demonstrate the immediate impact of the framework we propose in the analysis of firm behavior.

Consider a simple idea which will become the impetus for this paper.

At the start of time, an institution is instantiated in the sense that it ascribes an immutable rule to the principal. ${ }^{7}$ Since the immutable rule remains non-negotiable at all stages, this relationship is essentially a complete contract between the principal and the institution. ${ }^{8}$ Let us think of this as the level of the superstructure within which the principal-agent relationship, which we might consider as a proxy for the idea of a firm, plays out; and how we might define the borders of an institution. The immutable rule, though rigid in its structure, allows itself to be contextualized or 'tuned,' based on learning, and can hence either be made very precise or kept very general. We imagine the width of the borders of institutions to directly correlate with this precision. Ex post, the principal reports to the institution the level of opportunism, $v$, undertaken by the agent that she has been able to curb by controlling a process variable, $\phi$. The institution always accepts this, as long as it is below some maximum tolerance level $\left(\xi \phi^{\max }\right)$ and pays the principal: $y^{P}=\kappa W, r \leq \kappa \leq 1$, where $r W$ might be the reservation wage of the principal.

Meanwhile, the agent enters employment, or agrees to a contract with the principal, within a given institutional superstructure. By so doing he commits to a behavior that directly relates to a level of opportunism and accepts the fact that the principal will control his behavior. In this manner we rule out the possibility that, at the level of the superstructure, the institution cannot adjudicate on the performance of the principal or, indirectly, the behavior of the agent. The agent is himself the asset and his behavior determines value $V=\omega W, \omega \geq 1$ between them, which increases as the behavior improves. Without any ability for the principal to control behavior, the default rule remains, and a Nash bargaining solution yields the outcome. ${ }^{9}$ However, since we imagine the property rights

2. Persson (2002); Glaeser et al. (2004); de Haan (2007).

3. North (1990); Rodriguez-Pose/Storper (2006); Tabellini (2008); Williamson (2009).

4. La Porta et al. (2008).

5. Rodrik et al. (2004).

6. Rowley/Smith (2009).

7. The relationship of default and immutable rules to contract law is an interesting issue in the legal literature. Ayres and Gertner, as cited in Barnett (1992), suggest that 'default rules fill the gaps in incomplete contracts; they govern unless parties contract around them. Immutable rules cannot be contracted around; they govern even if the parties attempt to contract around them.' An immutable rule is not unlike what North (1991) calls a formal rule as the basis for an institution.

8. In the case of a sole owner, we may perhaps assist the imagination by thinking of the institution as the principal's alter ego.

9. Such an outcome has been analysed in Tirole (1999) and would yield $y^{A}=W(\omega-2 r) / 2$, $y^{P}=W(\omega+2 r) / 2$. 
over the agent's behavior not lying fully with either the agent or the principal, in this sense, their relationship is characterized by an incomplete contract. ${ }^{10}$

The purpose of this stylized example is to suggest how the generic idea of 'control' in contracts might be employed in understanding the structure of institutions and vice versa. This paper attempts to present a structuralist view on institutional design logic rather than make any attempt to endogenize bargaining between the agent and the principal directly, apart from in considering how information generated from such a process affects the institution.

Since the immutable rule is drawn from the preceding social norms, political traditions, and legal background rather than from the invocation or consideration of an equilibrium concept, the superstructure is therefore best not seen as a 'game form' in the sense it is used in the literature on implementation. This disjoint we see as necessary in making institutional rules primitive to the environment where institutions take shape and thus useful in explaining inertial change in institutions over time.

Therefore, in answering the question of what a firm is the position in this paper is that a firm is a conduit within a specific control process superstructure. This institutional superstructure provides and specifies an environment of specific control action that can be undertaken by a principal. This view is not meant to be a sensationalist departure in opinion from the literature on institutions, the nature of the firm, or contract theory, but rather a simple approach to understand the connections and relevance contained therein within an overarching and intuitive framework.

For Coase (1937), the firm is necessitated by a need to circumvent the cost of using the market, which is imagined here as the control process becoming markedly weaker for transactions that an institution engages in beyond its boundaries. To the extent that stronger control action is essential, the function must be brought within the scope of the superstructure. Williamson (1975) and Klein et al. (1978) suggest the role of asset specificity in exacerbating the potential for opportunism and holdup which can be thought of as modulating the strength of control action. Grossman/Hart (1986) aver that a firm is a collection of assets over which the principal has residual control rights which, in keeping with their insights, translates here as simply being a correlation between a given asset set and the strength of control action afforded to a principal. Interestingly, Hart/Moore (1990) suggest that the process of integration itself can be understood as emanating from the desire to strengthen the control process. The Aghion/Tirole (1997) framework of formal versus real authority can be understood as a formulation for how the control action within any control process superstructure can be tuned. Naturally, the message in these seminal contributions is more nuanced but the approach here allows understanding the relevance of the key message for any given application to the larger question of institutional structure.

We proceed in the following manner. Section 2 introduces the control process before it is developed in the context of a principal-agent relationship in Section 3. Section 4 then presents some illustrative extensions. First, in Section 4.1 we see how control action in a principal-agent relationship is structurally determined by the manner in which the institution is instantiated and thereby suggest how the approach is highly amenable to being contextualized to specific situations. Section 4.2 considers the role of knowledge and information in making the control action specific with soft knowledge or expert insight.

10. However, with perfect control - for example, when the agent is a robot or slave - trivially we have $y^{P}=\kappa W$ and $y^{A}=0$. 


\section{THE CONTROL PROCESS AS A SUPERSTRUCTURE}

To understand our motivation for a structuralist view, it is useful to begin by considering a stochastic dynamic environment where a steady state can be defined by a single parameter $\phi(t)=\phi^{*}$. It is perhaps helpful to think of this parameter as being designed into a mechanism or, alternatively, as expected by a set of experts. To focus thought, let us imagine a principal-agent set-up where this parameter describes the degree of deviation, $y^{*}$, which an agent can theoretically sustain from a desired outcome, $x^{*}$, as defined by a set of principals within a given social, economic, or political institutional environment.

In a real-world environment, if we believe that this deviation can be understood to follow a process such as $y_{t}=\beta_{0}+\beta_{1} x_{t}+\beta_{2} x_{t-1}+\beta_{3} y_{t-1}+\varepsilon_{t}$, then it is logical to assume that the equilibrium solution to the problem would yield the steady-state constant $y^{*}-x^{*}=\phi^{*}$ with $\beta_{1}=\beta_{2}=\beta_{3}=1$. It is illustrative to understand this set-up as an error-correction model such as:

$$
\Delta y_{t}=\beta_{0}+\beta_{1} \Delta x_{t}+\left(\beta_{3}-1\right)\left(y_{t-1}-x_{t-1}\right)+\varepsilon_{t},
$$

where $\Delta y_{t}$ is the error correction and $y^{*}-x^{*}=\phi^{*}$ describes the long-term equilibrium condition. ${ }^{11}$

In a static framework, a number of perspectives exist in the literature, focusing on interesting aspects of the contract existing between the principal and the agent; indeed, a host of variables may matter, including the relevance, value, and symmetry of information, the scope for strategic behavior, and the timing of decisions. However, in a dynamic and stochastic setting, the cost of making $\left|\phi^{*}\right|=0$ may be known to be infinite due to the knowledge that, in the real world, there always exists a steady-state error. We consider the source of this knowledge to be that all forms of institutions in any given society are expected to be imperfect and thus incapable of eradicating all error in the social processes. ${ }^{12}$

Therefore, rather than focusing on a specific nuance of this setup, we step back and explore the roots of structural rigidities. Specifically, the setup I am proposing suggests how control process engineering might be used to model a superstructure to investigate relationships between principals and agents and perhaps other entities that are relevant but which fall outside the purlieus of most investigations such as social capital, cultural context, and path dependency in social outcomes. The paper thereby also suggests how control process theory can be seen as providing a useful and unifying structural representation for a wide variety of dynamic equilibrium processes.

The traditional application of control process engineering is for designing the automatic control of simple as well as highly complex systems in order to achieve desired targets. ${ }^{13}$ The type of system itself is of less importance than its dynamic behavior, so

11. For an ECM to work correctly, naturally we would also need to ensure that $\left(y_{t}-x_{t}\right)$ is $I(0)$ and that $\beta_{3}<1$.

12. This is hardly a contentious claim. In economics, explanations on the existence of firms often draw on the observation that they combat regulation and avert the costs of marketbased exchange. In politics, regime characteristics and constitutions are seen as describing the scope of government in society and thereby leaving differing degrees of residual control with political entities. In sociology, the role of trust and social capital has been shown to clearly impinge on the functioning of government and markets.

13. The typical use of control engineering - and its hallmark, PID controllers - varies from designing robotic responses to fluid mechanics, industrial engineering, aerospace engineering, and so on. It is employed in designing and analysing a variety of systems that require careful 
the methodology has wide applicability, ranging from thermostats to cruise control and auto-pilot systems. The use of feedback loop systems and control processes in the social sciences is rare, but not without exception. Robinson (2007), for example, provides a useful survey of its use in sociology.

The process has some general characteristics such as the presence of a plant where the process actually takes place. This could be thought of as an arena for applications in the social sciences where agents take action, often collaboratively, to enable an outcome. Certain input variables are provided to the plant where random disturbances may impact the process before an output is produced. The values of these input variables naturally depend on what is instrumentally desired by the controller which we may consider to be the principal at an institution. In an engineering system, the controller sends a signal using very low energy to the plant, such as a preset speed or course for a vehicle or aircraft. The plant then employs higher quantities of energy in achieving the target (viz. brakes, throttle, trim, and so on). The output is then metered against the target in the calculation of corrective signals for the next round. We can easily think of a social science system where the controller (principal) sends a signal of desires using reward structures to the arena where actual action is taken by agents and then the controller receives feedback on the output from a measurement mechanism (which could be thought of as a poll, a consultant, a computer or even a media report) in determining its corrective measures. As we shall see, the process of feedback in this methodology is one of the key aspects that suggests its promise in understanding social scientific systems.

\section{CONTROLLING AGENT BEHAVIOR}

In control process engineering, PID (or Proportional-Integral-Derivative) tuning theory clearly suggests how a complex system dealing with movement to and from a theoretical ideal 'setpoint' may be manipulated and controlled, and it is thus rather useful in analysing the behavior of an agent when seen as an entity being modulated by the institution rather than the principal directly.

$\phi(t)$ is the process that can be seen as capturing the problem of incentive incompatibility. It is the observed deviation of each period of the agent, emanating from her actions, from the setpoint in the institutional environment, and is clearly a variable that the institution (principal) monitors. The institution's controller is a corrective instrument that produces an output that motivates movement of the agent within a defined dimension. Adjusting the corrective output determines the desired rate of adjustment. A higher output level corresponds with a desired goal of faster adjustment.

\subsection{Proportional control}

Let us start by imagining that, if an agent's actions are not entirely aligned with the desires of the institution, she engages in some form of opportunistic behavior, $v(t)$, which increases her wealth. If at time $t$ the corrective output from the institution is $R(t)$, this enables the agent to engage in $v(t)>0$ and deviate from the optimally expected action by some $\phi(t)$ units. So $v(t)=\xi \phi(t)$ where $\xi>0$. The institution

monitoring and controlling of specific processes. While books and journals dealing with control theory abound, for more on PID control and a very detailed analysis of aspects of control theory, Dean/Wellman (1991) is a good place to start. 
would continually output some form of corrective response as long as $\phi(t)>\phi^{d}$ where $\phi^{d}$ is the tolerable level of deviation in observed behavior that the agent is ideally permitted by the institution. ${ }^{14}$

Therefore, if at time $t$ the corrective output from the institution is $R(t)$ and this enables the agent to deviate from the optimally expected action by some $\phi(t)$ units, this process, in the terminology of control process engineering, is said to have a gain that measures how much $\phi(t)$ changes when the controller's output changes. Let us call this the institution's leverage as defined by $L=\phi(t) / R(t)$. So if $R(t)$ is understood as the institutionally feasible control level, then $L$ can be seen as the agent's perception of this baseline constraint on $v(t)$.

Control in this relationship works through the process

$$
\phi(t)=L \cdot R(t) .
$$

So from Equation (1) we can infer that the lower the agent's perception, or the actual level, of the control level which is output by the institution, the higher is the deviation.

However, the control mechanism of the institution itself has an intrinsic efficiency, $\chi$, that determines its output at any given time, $t$, according to

$$
R(t)=\chi\left(\phi_{\max }-\phi(t)\right),
$$

where $\phi_{\max }$ is a constant that describes the maximum distance that the agent can deviate when the level of corrective output the institution can exert is at its highest possible value. So the quantity $\left(\phi_{\max }-\phi(t)\right)$ that enters the institution's observation mechanism as an input gains strength by some factor $\chi$ before it is output to the process as the corrective control output $R(t)$.

The essential significance of Equation (2) is that it accounts for differing abilities across institutions to ameliorate situations of incentive incompatibility using their existing control structures.

This control equation can be rewritten as

$$
R(t)=\chi\left(\phi^{d}-\phi(t)\right)+R_{B}
$$

where $\phi^{d}$ is achieved when $\phi(t)$ equals the setpoint. $R_{B}=\chi\left(\phi_{\max }-\phi^{d}\right)$ is a constant called the bias, which represents the control output level required to maintain $\phi(t)$ at its desired level in the absence any exogenous shock or disturbance to the process. Equation (3) shows how the institution's output might be computed as a result of the error between the process variable at the theoretical goal.

This form of control behavior is called a proportional control system due to the leverage coefficient. The greater the error, the greater the control output, and as long as error remains, the institution will need to continue to output a corrective level of control.

\subsection{Integral control}

In the presence of disturbances to the institutional environment, the proportional corrective output by the institution will leave a steady state error in $\phi(t)$. The agent perceiving a proportional-only institutional control structure could in fact increase $v(t)$ by

14. It is logical that this response should be a function of the degree of error. 
undertaking strategic behavior that increases the level of disturbance as perceived by the controller. Proportional control can lead to persistent problems. To see this, simply assume that $L=\chi=R_{B}=1 .{ }^{15}$ If the tolerable deviation goal requires $\phi^{d}=3$ and $\phi(t)=2$, this implies a deviation of $\phi^{d}-\phi(t)=1$. The institution's control structure will amplify the control level to 2 according to Equation (3). However, since the output level of 2 will in turn cause $\phi(t)$ to remain at 2 , the controller will make no further changes and the error in deviation will remain at 1 .

An integral corrective can solve this problem by generating a control output that is proportional not to the present deviation, but to accumulated deviations. With proportional control, the institution's control mechanism uses the control output level to manage the degree to which the agent engages in opportunist behavior (that is to say, the distance the agent deviates from the institution's setpoint). The objective remains to readjust the distance an agent deviates whenever a disturbance causes significant change in $v(t)$. However, for an institution with memory of an agent's opportunistic behavior,

$$
\phi(t)=\int L \cdot R(t) d t
$$

which shows that deviation depends not only on the level of perceived control output but also on the length of time the control is applied and, thus, in the sense of integral action, the institution's control mechanism is more effective. This form of control structure adopted by an institution will not generally admit a steady-state error.

\subsection{Derivative control}

Consider the situation where the agent's deviation decreases until its desired value is reached and the control output ceases. If, however, the control mechanism and the deviation process follow a function such as (4) then the agent's deviation would grow unbounded and the steady-state error would explode. In combination, however, the Proportional-Integral control structure would be more effective in controlling the agent but it might take too long to compensate for a change that alters the environment. Here, a derivative corrective effort by the control structure would be useful, since it would generate a corrective control based on the time derivative of the agent's deviation. Derivative control is a very sophisticated control procedure and would be sensitive even to measurement error. In a perturbed system, such a method control would generate one large corrective control action to begin eliminating deviation immediately. If, for instance, a surge in $v(t)$ occurs at time $t_{0}$, the time derivative function of the agent's deviation would demonstrate an even larger surge at $t_{1}$ since the derivative of a step-function is an even more pronounced impulse function. Conversely, closer to the target, this sort of control would slow the output of control effort down. In short, it is a corrective mechanism that only a more informed institution could employ. The problem with derivative control is that it is sometimes too crude on its own without integrative and proportional correctives and would make for a poor control instrument. Integrative correctives are susceptible to the problem (known as the closed-loop problem) that a large leverage will allow aggressive corrective control usage to transform the agent's deviation into another large deviation in the opposite direction, which may be counterproductive. The institution is then likely

15. Essentially this is saying that the leverage is 1 , so that any control output level will cause an identical adjustment in deviation by the agent. 
to suffer from the problem of oscillating between maximum threat output and minimum threat output. Proportional correctives would be unlikely to cause this, but on their own they are prone to bias.

While any particular institution may be in a position to employ a PI, P only, I only or PD controller, the unified form of an electorate PID control structure then is:

$$
C(t)=P \cdot e(t)+I \cdot\left(\int_{0}^{t} e(t) d t\right)+D \cdot\left(\frac{d}{d t} \cdot e(t)\right),
$$

where $C(t)$ is the control output at time $t$ and $P, I$ and $D$ are known as the tuning constants for the institution. $e(t)$ is simply the difference between the setpoint and the process variable or $e(t)=\phi^{d}-\phi(t)$.

\section{CONTROL THEORY IN PRACTICE}

\subsection{A context-sensitive institutional approach to the principal-agent relationship}

A control process superstructure is arguably useful in describing institutional parameters rather well, since the manner in which control action can feasibly be tuned delimits an institution's boundaries. As such, control process superstructures can help contextualize institutions to particular environments too. As an illustration, let us examine the principal-agent relationship in a simple and static model that highlights the role of control action by the principal within a given and instantiated institutional environment and contextualize the entire problem.

The agent plans opportunistic behavior based on control in a given institutional environment. Therefore we have:

$$
v=\eta+(c+\varepsilon)
$$

where $v$ is the opportunist behavior which can assume negative or positive values, $\eta$ is the 'effort' that the agent puts into shirking, $c$ is a control output from the principal that depends on the institutional policy that the principal is operating with, and $\varepsilon$ exists to capture discrepancies in the control action of the control policy. The principal and agent enter into a contract with value $R$ that implicitly or explicitly specifies that the reward assigned to the agent, $r$, is based on an ex ante expectation of $v$. The institution then produces output $y$, such that

$$
y=\bar{y}-R+v-\varepsilon \text { or equally } y=\bar{y}-r+(v-E(v))-\varepsilon .
$$

The timing of the process is that the institution is instantiated, and only then do the principal and agent both see its value as $(\bar{y}-r)$. A contract is then offered, based on $E(v)$. The agent puts in his desired level of shirking and the value of the disturbance in the output process $(\varepsilon)$ becomes known. The principal then uses the institutional parameters to determine $c$ and concomitantly $\varepsilon$ becomes known too. Finally, output and opportunistic behavior become evident.

This timing captures the ideas that institutional as well as agent quality can play a role in the contract and that the institutional parameters on policy determine control action by principals with this information drawn from a larger context rather than the institution itself. Socially sensitive and contextualized knowledge (or 'soft' knowledge) becomes important. 
The control then becomes a means to smooth out the intrinsic propensity for opportunism and institutional inertia. Therefore

$$
E(v)=E(v \mid(\bar{y}-r)) \text { and } E(v)=E(c \mid(\bar{y}-r)),
$$

which implies that

$$
y=\bar{y}-r+c-E(c \mid \bar{y}-r))+\eta+\epsilon-\varepsilon .
$$

Note that the control action falls from the institutional parameters which the principal takes as given. The institution is thus truly instantiated - knowingly or unknowingly taking soft knowledge or stereotypes - as a mapping such as

$$
c \leftarrow \Lambda[(\bar{y}-r), \eta, \varepsilon] .
$$

The social context evaluations of institutional outcome proceeds according to some trade-off:

$$
E(T(v, y))=E\left((v-\widehat{v})^{2}+\alpha(y-\widehat{y})\right) / 2
$$

where the terms $\widehat{v}$ and $\widehat{y}$ suggest the role for very contextual judgments on how well an institution need perform and what level of opportunism is socially acceptable. This, said in perhaps less interesting terms, can be restated as the contextual emphasis on the trade-off between a market and a social tradition.

The parameters for control action come from institutional instantiation in this formulation and therefore occur before the institutional quality $(\bar{y}-r)$ is actually realized. It is a 'tuned' combination that the principal must assume, of the form:

$$
c=\Lambda+\Lambda_{(\bar{y}-r)}(\bar{y}-r)+\Lambda_{\eta} \eta+\Lambda_{\varepsilon} \varepsilon
$$

and the agents in the society will simply proceed, thinking that

$$
E(c \mid(\bar{y}-r))=\Lambda+\Lambda_{(\bar{y}-r)}(\bar{y}-r) .
$$

In equilibrium, then,

$$
\begin{gathered}
v=\Lambda+\Lambda_{(\bar{y}-r)}(\bar{y}-r)+\left(\Lambda_{\eta}+1\right) \eta+\Lambda_{\varepsilon} \varepsilon+\epsilon \\
y=(\bar{y}-r)+\left(\Lambda_{\eta}+1\right) \eta+\left(\Lambda_{\varepsilon}-1\right) \varepsilon,
\end{gathered}
$$

and the principal must learn to adapt when selecting efficient control action by considering

$$
\begin{gathered}
E(T(v, y))= \\
E\left(\left(\left(\Lambda+\Lambda_{(\bar{y}-r)}(\bar{y}-r)+\left(\Lambda_{\eta}+1\right) \eta+\Lambda_{\varepsilon} \varepsilon+\epsilon\right)-\widehat{v}\right)^{2}+\alpha\left(\left((\bar{y}-r)+\left(\Lambda_{\eta}+1\right) \eta+\left(\Lambda_{\varepsilon}-1\right) \varepsilon\right)-\widehat{y}\right)\right) / 2 .
\end{gathered}
$$

Setting the first-order conditions of this with respect to the institutional parameters condition equal to zero yield the following three lemmas on the principal's behavior pertaining to control action:

Lemma 1

$$
\Lambda=\bar{v} \text { and } \Lambda_{(\bar{y}-r)}=0
$$


which says that the efficient control action is highly context-sensitive, depending solely on the society's acceptance of opportunism and not on the intrinsic value of the institution or the preference for institutional performance.

Lemma 2

$$
\Lambda_{\eta}=-1
$$

which essentially says that the value of control action by the principal is in nullifying the effort undertaken by the agents to shirk and

Lemma 3

$$
\Lambda_{\varepsilon}=\alpha /(1+\alpha)
$$

which says that control action to nullify disturbances to the institution's output is context sensitive, and that, if the preference is higher on output, then such disturbances will be more actively managed.

The control action behavior by the principal within an instantiated institution environment is therefore:

Theorem 1(a): Contextualized control action behavior

$$
c=\widehat{v}-\eta+(\alpha /(1+\alpha) \varepsilon)
$$

which results in contextualized levels of opportunism and institutional quality

Theorem 1(b): Contextualized levels of opportunism

$$
\widetilde{v}=\widehat{v}+\varepsilon+(\alpha /(1+\alpha) \varepsilon)
$$

Theorem 1(c): Contextualized institutional output behavior

$$
\tilde{y}=(\bar{y}-r)+\varepsilon-(\alpha /(1+\alpha) \varepsilon) .
$$

These results suggest that control action error by the principal plays a role in ameliorating the contextualized levels of opportunism and institutional output in conjunction with the relative emphasis placed by the agent and the institution on context sensitivity.

\subsection{From fuzzy control to specific control correctives}

In order to highlight the fact that contextual sensitivity is a key benefit of this approach, consider now how this control structure might be informed by expert opinion and other real-world information by employing fuzzy-logic control structures. ${ }^{16}$ To motivate this discussion, it is useful to think of the degree of completeness in a contract to correlate with the crispness with which a tuning equation such as Equation (5) can be specified. A complete contract in a frictionless world is therefore one where the process variable always stays at the setpoint. However, this assumes that the ex ante and ex post desired levels of agent deviation are zero. This may not always be optimal in a dynamic environment where the institution wishes to encourage initiative and

16. This is meant to be an example. For fuller treatments, see Russo/Jain (2000) and Reznik (1997). 
innovation by the agent ${ }^{17}$ or where the setpoint itself needs constant adjustment. A complete contract becomes less desirable and incompleteness more agreeable. This can be seen with the control process superstructure as making the contract contextually sensitive and relying more on imperfect or fuzzy information rather than perfect or crisp information.

Fuzzy control of agent behavior is even more relevant than a model that approaches the problem from a frequentist probabilistic framework, simply because the former allows a subjective perspective which mimics more precisely how an institution is likely to behave. In researching the types of firms, a researcher could seek the advice of experts or those with experiential knowledge and this would then enable providing the relative values of the PID components and comparing across systems.

Consider an example of a PI control mechanism as the superstructure at an institution:

$$
C(t)=L \cdot \Delta e+L \cdot\left(\frac{\Delta t}{T_{i}}\right) \cdot e .
$$

An experienced principal at an institution or perhaps even third-party experts can be seen as expert tuners of this control structure. Their heuristic knowledge, intuition, or private information may guide them on how to appropriately tune the control structure and thus how to effectively package the total control effort in a given period. ${ }^{18}$

Such experts that have observed a shirking agent deviating some distance from the desired behavior are likely to suggest a level of control effort based on current and past deviations of the agent, not in contractually quantifiable terms but, rather, in linguistic ones only. They might review the agent as 'somewhat lazy' or favor a 'firm degree' of corrective control. This can be operationalized using fuzzy-logic control.

Fuzzy logic is the superset of set theory. Rather than the clean distinctions of set membership that Venn diagrams depict, fuzzy sets are better seen as smudges, such that the center of the set indicates rather clear membership, but at the outer edges the probability of membership to that set diminishes. Specifically, set membership is a function bounded by 0 and 1 , where 0 represents a complete absence of membership to a set and 1 represents a complete membership to a set. The determinations made on increasing or decreasing degree of membership to a set can thus be made subject to opinions, by giving the 0 to 1 scale a rich ordinal interpretation, which is something not possible with probability theory. Membership functions can take the functional form of a trapezoid or an isosceles triangle, although smooth membership functions are also possible.

Both inputs of the PI controller in Equation (6) are then normalized such that they can be represented on the same scale when fuzzified into categories such as Zero $(\mathrm{O})$, Small (S), Medium (M), and Large (L), as shown in Figure 1. Then a control

17. As in the literature in contract theory on project selection, such as Holmstrom/Milgrom (1987).

18. As a matter of fact, one could even consider the agent as providing information directly on tuning the system more effectively. One way of seeing how such information might be used as fuzzy feedback is by seeing the model in Ross (1973). The principal - agent model there presents a Pareto-efficient fee schedule based on $\max E\{U[\omega-f]+\lambda G[f]\}$ which gives $U^{\prime}[\omega-f]=\lambda G^{\prime}[f]$ and where $\lambda$ is the weight on the agent's utility. That parameter would scale the relevance of the feedback received from the agent in tuning, but more fundamentally, the fee-schedule $f$ could be conceived of being dependent on states of the world, $\Theta$, that are themselves a function of control output, $C$, and control error and thus a pay-off to the agent that is written as $\omega(\phi, \Theta)$. 


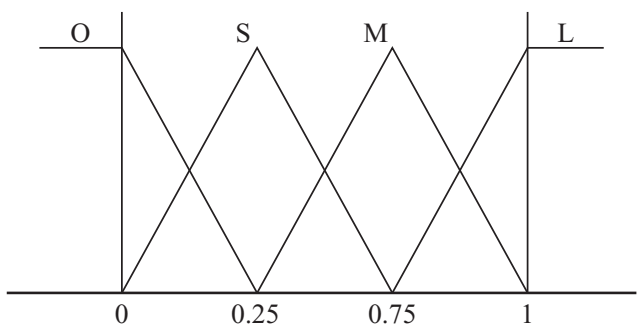

Figure 1 An isosceles membership function

\begin{tabular}{cc|c|c|c|c} 
& \multicolumn{5}{c}{ Proportional control } \\
& & $\mathrm{O}$ & $\mathrm{S}$ & $\mathrm{M}$ & $\mathrm{L}$ \\
\cline { 2 - 6 } $\begin{array}{c}\text { Integrative } \\
\text { control }\end{array}$ & $\mathrm{O}$ & $\mathrm{O}$ & $\mathrm{S}$ & $\mathrm{S}$ & $\mathrm{M}$ \\
\cline { 2 - 6 } & $\mathrm{S}$ & $\mathrm{S}$ & $\mathrm{S}$ & $\mathrm{S}$ & $\mathrm{M}$ \\
\hline & $\mathrm{M}$ & $\mathrm{S}$ & $\mathrm{S}$ & $\mathrm{M}$ & $\mathrm{M}$ \\
\hline & $\mathrm{L}$ & $\mathrm{M}$ & $\mathrm{M}$ & $\mathrm{M}$ & $\mathrm{L}$
\end{tabular}

\section{Figure 2 PI Control algorithm matrix}

algorithm such as the one shown in Figure 2 is devised that relates the control action to inputs.

Since this control algorithm takes the form of a matrix, a PID controller requires a 3-D control algorithm matrix. The control algorithm would of course have to be based on rich and contextual information from experts. Thereafter, group membership rules, $\gamma,{ }^{19}$ would have to be devised for the control action such that it followed a fuzzy-set logic such as:

$$
\begin{aligned}
& M I N: \Upsilon_{A \cap B}(y)=\Upsilon_{A}(y) \cap \Upsilon_{B}(y) \\
& M A X: \Upsilon_{A \cup B}(y)=\Upsilon_{A}(y) \cup \Upsilon_{B}(y)
\end{aligned}
$$

Now a defuzzification step needs to be undertaken back from expert insight to a quantitative output for use in the control structure. A straightforward approach for doing this is to assume that we have some values for the I and P control inputs such as 0.1 and 0.5 . This would suggest that Integrative Control belongs either to $\mathrm{O}$ or to $\mathrm{S}$ and the Proportional Control belongs to either $\mathrm{S}$ or $\mathrm{M}$, and thus

$$
\begin{gathered}
\Upsilon_{\mathrm{o}}(e)=0.9 ; \Upsilon_{s}(e)=0.1 \\
\Upsilon_{s}(\Delta e)=0.5 ; \Upsilon_{s}(\Delta e)=0.5
\end{gathered}
$$

19. Bounded between 0 and 1 , such as a CDF stretched to represent the level of certainty the experts have. 


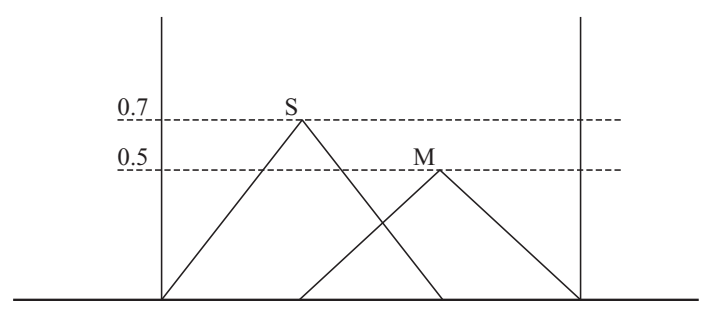

Figure 3 Defuzzified and adjusted

Finally, to quantify the control action output for the entire PI superstructure, the membership functions can be used to suggest:

$$
\begin{aligned}
& e \leftrightarrow \Delta e: \Delta C_{o}=S=\operatorname{MIN}\left[\Upsilon_{o}(e), \Upsilon_{s}(\Delta e)\right]=0.5 \\
& e \leftrightarrow \Delta e: \Delta C_{o}=M=\operatorname{MIN}\left[\Upsilon_{o}(e), \Upsilon_{m}(\Delta e)\right]=0.5 \\
& e \leftrightarrow \Delta e: \Delta C_{s}=S=\operatorname{MIN}\left[\Upsilon_{s}(e), \Upsilon_{m}(\Delta e)\right]=0.1 \\
& e \leftrightarrow \Delta e: \Delta C_{s}=S=\operatorname{MIN}\left[\Upsilon_{s}(e), \Upsilon_{s}(\Delta e)\right]=0.1,
\end{aligned}
$$

which suggests that the control superstructure would employ small and medium-sized fuzzy-logic correctives with magnitudes of 0.7 and 0.5 . However, we still require an estimate for the leverage of the two inputs, which then gives us the desired control action output. One of the most common ways of proceeeding is to find the center of gravity of the now adjusted figure (shown in Figure 3) obtained from the relevant membership functions.

The numerical control corrective based on fuzzy feedback is then:

$$
\Delta C=\frac{0.25 \cdot 0.7+0.75 \cdot 0.5}{0.7+0.5}=0.46
$$

It is worthwhile, at this juncture, to reflect on the benefit of such a perspective. We believe that without a methodical evaluation of context-sensitivity and subjective points of departure, to our detriment, our ability to assess how feasible institutions differ from ideal ones is truly conscribed. Consider the discussion of second-best institutions in Rodrik (2008). These are institutions developed with a localized context in contrast to the set of first-best institutions that result from the traditional resolution of property rights provision concerns and managing the course of macroeconomic stabilization programs. The need for second-best institutions is an acknowledged issue in modern development economics and development policy. And perhaps it is not an insurmountable problem. An analysis of second-best institutions need not be different from that of the set of first-best ones, albeit with a fuzzy interpretation on the features of the first-best based on locally-relevant information. The resulting recommendations are then more likely to pass the test of context sensitivity, and, consequently, more likely to be successfully adopted than the boiler-plate advice often given and routinely denounced in retrospect for being insensitive or inappropriate.

\section{CONCLUSION}

As far back as 1934, Commons had suggested the role of collective action and collective control in institutional economics. So, in employing control process theory, what 
we propose in this paper is not a call for a radical shift in thinking about institutions. Instead, it is an acknowledgment that one of the key concerns about formal studies on institutions is that they neglect the relevance of the specific contexts within which they are meant to function. To tackle this problem, we have developed a framework based on control theory to incorporate context sensitivity in a methodical manner. We acknowledge that this is hardly a satisfactory manner of introducing context sensitivity to a research project when the intention is to carefully unpack the nature of the context in order to gain a rich understanding of it. However, we believe it is a promising method of fine-tuning an analysis for context sensitivity, and, as such, it also allows for collaborative efforts in analysis where deep qualitative insights can have a valuable input to formal characterization.

We used the idea of an institutional superstructure in this paper for contextualizing a firm. We maintain that it is illustrative to formally think about the general idea of control in contractual relationships by parsing it as being reliant on primitive top-down sources. We consider an institution as a superstructure that frames a principal-agent relationship by providing an immutable rule, which is unique to the context. This immutable rule is non-negotiable, but allows considerable scope for manipulation through information. Among the numerous avenues open for future research using control theory, this is a rather interesting one; we do not consider the symmetry of this information in our discussion of fuzzy learning in this paper and its impact on the efficacy of the behavior of the entire system. We also do not consider the impact of changing setpoints, tolerance for opportunistic behavior, costs for recursively tuning the system, and competition among agents. To the extent that these or any other issues provide useful intuition on the nature of institutions, we believe they are worthwhile exercises. It is important to reiterate here that this approach is not meant to reinvent the wheel but, rather, to understand how it functions as part of the vehicle it is attached to.

It is true that this approach of a superstructure provides a degree of structural determinism. This, we admit, is intentional, in that there is a nagging sensation that the primacy of institutions is not indefensible when one considers two simple facts. Both heterodox and neoclassical institutional economists suggest the relevance of institutions in framing market exchange. In our set-up, the immutable rules of an institutional superstructure are, therefore, deliberately imagined as more enduring than the contractual relationships that firms represent. A pithy observation that North makes is that institutions can be understood as constraints and rules that can only be changed incrementally over time and he even suggests that the source of this change might be an entrepreneur responding to incentives that have been shaped by his environment (North 1990). Further, with regard to such change, Williamson (2000) suggests how the order of change is progressively slower for social institutions than it is for political and economic ones. Given that social and economic institutions do not live in isolation, this inertial change in social institutions should logically provide a degree of structural determinism to economic exchange. The set-up in this paper caters to this view rather well.

\section{REFERENCES}

Aghion, Phillipe, Tirole, Jean (1997): Formal and real authority in organizations, in: Journal of Political Economy, 105(1), 1-29.

Barnett, Randy E. (1992): The sound of silence: default rules and contractual consent, in: Virginia Law Review, 78, 821-912.

Che, Jiahua, Facchini, Giovani (2009): Cultural differences, insecure property rights and the mode of entry decision, in: Economic Theory, 38(3), 465-484. 
Coase, Ronald H. (1937): The nature of the firm, in: Economica, 4, 386-405.

Commons, John (1934): Institutional Economics: Its Place in Political Economy, New York: Macmillan.

Dean, T., Wellman, M. (1991): Planning and Control, San Francisco, CA: Morgan Kaufmann Publishers.

de Haan, Jakob (2007): Political institutions and economic growth reconsidered, in: Public Choice, 131(3), 281-292.

Hart, Oliver, Moore, John (1990): Property rights and the nature of the firm, in: The Journal of Political Economy, 98(6), 1119-1158.

Holmstrom, Bengt, Milgrom, Paul (1987): Aggregation and linearity in the provision of intertemporal incentives, in: Econometrica, 55(2), 303-328.

Glaeser, Edward L., La Porta, Rafael, Lopez-de-Silanes, Florencio, Shleifer, Andrei (2004): Do institutions cause growth?, NBER Working Paper Series, Working Paper 10568.

Grossman, Sanford J., Hart, Oliver (1986): The costs and benefits of ownership: a theory of vertical integration, in: Journal of Political Economy, 94(4), 691-719.

Klein, Benjamin, Crawford, R.G., Alchian, A.A. (1978): Vertical integration, appropriable rents, and the competitive contracting process, in: Journal of Law and Economics, 21, 297-326.

La Porta, Rafael, Lopez-de-Silanes, Florencio, Shleifer, Andrei (2008): The economic consequences of legal origins, in: Journal of Economic Literature, 46(2), 285-332.

North, Douglas (1990): Institutions, Institutional Change and Economic Performance, Cambridge, UK: Cambridge University Press.

North, Douglas (1991): Institutions, in: Journal of Economic Perspectives, 5, 97-112.

Persson, Torsten (2002): Do political institutions shape economic policy?, in: Econometrica, 70(3), 883-905.

Reznik, Leon (1997): Fuzzy Controllers, Oxford and Boston: Newnes.

Robinson, Dawn T. (2007): Control theories in sociology, in: Annual Review of Sociology, 33, 157-174.

Rodríguez-Pose, Andrés, Storper, Michael (2006): Better rules or stronger communities? On the social foundations of institutional change and its economic effects, in: Economic Geography, $82(1), 1-25$

Rodrik, Dani (2008): Second-best institutions, in: American Economic Review, 98(2), 100-104.

Rodrik, Dani, Subramanian, Arvind, Trebbi, Francesco (2004): Institutions rule: the primacy of institutions over geography and integration in economic development, in: Journal of Economic Growth, 9(2), 131-165.

Ross, Stephen A. (1973): The economic theory of agency: the principal's problem, in: The American Economic Review, 63(2), 134-139.

Rowley, C.K., Smith, N. (2009): Islam's democracy paradox: Muslims claim to like democracy, so why do they have so little?, Public Choice, 139(3-4), 273-299.

Russo, Marco, Jain, Lakhmi C. (2000): Fuzzy Learning and Applications, Boca Raton: CRC Press.

Tabellini, Guido (2008): Presidential address: institutions and culture, in: Journal of the European Economic Association, 6(2/3), 255-294.

Tirole, J. (1999): Incomplete contracts: where do we stand?, in: Econometrica, 67(4), 741-781.

Williamson, Claudia R. (2009): Informal institutions rule: institutional arrangements and economic performance, in: Public Choice, 139(3/4), 371-387.

Williamson, Oliver E. (1975): Markets and Hierarchies: Analysis and Antitrust Implications, New York: Free Press.

Williamson, Oliver E. (2000): The new institutional economics: taking stock, looking ahead, in: Journal of Economic Literature, 38(3), 595-613. 